\title{
Corpus
}

$23 \mid 2022$

Corpus et données en morphologie

\section{Les personnages de théâtre ont-ils un style? Recherche outillée sur un corpus théâtral trilingue}

Do Theatrical Characters Have a Style? Tool-based Research on a Trilingual Theatrical Corpus

Marc Vandersmissen

(2) OpenEdition

Journals

Édition électronique

URL : https://journals.openedition.org/corpus/6680

DOI : $10.4000 /$ corpus. 6680

ISSN : $1765-3126$

Éditeur

Bases; corpus et langage - UMR 6039

Référence électronique

Marc Vandersmissen, «Les personnages de théâtre ont-ils un style ? Recherche outillée sur un corpus théâtral trilingue », Corpus [En ligne], 23 | 2022, mis en ligne le 18 février 2022, consulté le 05 mars 2022. URL : http://journals.openedition.org/corpus/6680 ; DOI : https://doi.org/10.4000/corpus.6680

Ce document a été généré automatiquement le 5 mars 2022.

(c) Tous droits réservés 


\title{
Les personnages de théâtre ont-ils un style? Recherche outillée sur un corpus théâtral trilingue
}

\author{
Do Theatrical Characters Have a Style? Tool-based Research on a Trilingual \\ Theatrical Corpus
}

Marc Vandersmissen

\section{Introduction}

Ces dernières décennies, l'évolution des outils informatiques a profondément modifié la recherche dans le domaine des studia humanitatis. Cette transformation s'est opérée à la fois sur les techniques de traitement (par exemple, de la lexicométrie à la logométrie), sur les objets (types de sources, natures des corpus, genres littéraires ou non), et sur l'essence même de nos études (développement de la statistique textuelle, traitement automatique du langage, humanités numériques). La stylistique a bénéficié, elle aussi, de ces avancées et se voit aujourd'hui évoluer vers la stylistique dite " outillée ». Cette étape illustre à quel point cette discipline et ses méthodes sont désormais inextricablement liées ${ }^{1}$. Ainsi, la notion de style a-t-elle été étendue à de nouveaux objets textuels, non plus seulement littéraires. Citons comme exemples les didascalies au théâtre ou bien le discours scientifique ${ }^{2}$. De nouveaux marqueurs stylistiques ont été mis au jour, entre autres, grâce aux méthodes statistiques, comme les stylèmes ou les motifs multidimensionnels ${ }^{3}$. Enfin, la définition même de style d'un texte semble ne pouvoir se fixer définitivement qu'au sein de son corpus de référence. C'est dans ce cadre théorique novateur et stimulant que je propose de réfléchir sur la pertinence d'appliquer le concept de style aux personnages de théâtre à l'aide des outils de la statistique textuelle. Je cherche donc non seulement à questionner la définition même de style, mais aussi à mieux comprendre par ce biais les processus de création d'une œuvre théâtrale. Pour rencontrer cet objectif, je tenterai de répondre aux questions suivantes : peut-on recourir à la notion de style pour un personnage 
fictif? Plusieurs personnages d'une même pièce peuvent-ils avoir les mêmes caractéristiques stylistiques? Ces particularités textuelles sont-elles suffisamment caractérisantes pour définir un style à part entière à ce niveau très local de texte ? Comment définir ce qui relèverait du style ou du discours (types de personnages), du registre de communication (collectif vs individuel, statuts sociaux différents...) ou du mode de locution (monologue vs dialogue, versification...) ? Pour ce faire, je fonderai ma réflexion sur un corpus composé de tragédies d'Euripide, de Sénèque et de Corneille. Avoir recours à des auteurs d'époques et de langue différentes permet les comparaisons, mais se limiter à un genre commun facilite la généralisation du propos. D'abord nous allons nous interroger sur la nature des rôles des personnages. Ensuite, nous chercherons à déterminer si ces unités peuvent avoir un style. Enfin, nous questionnerons le rapport entre étude du discours et stylistique.

\section{Le rôle d'un personnage est-il un texte?}

Pour étudier le concept de style appliqué aux personnages de théâtre, il convient en premier lieu de définir sur quels supports textuels travailler. Il est en effet nécessaire de dépasser le commentaire stylistique d'une réplique spécifique et de traiter l'ensemble des interventions sur scène d'un personnage comme une unité individuelle. De cette manière, il est possible de comparer les personnages ensemble au sein d'une même œuvre ou bien du même auteur. Aujourd'hui, les logiciels de traitement automatique de la langue permettant d'interroger des corpus annotés morphosyntaxiquement offrent, grâce à l'utilisation de balises spécifiques, de nouvelles possibilités de création de corpus ${ }^{4}$. Ainsi, il est possible de découper facilement une pièce de théâtre, par exemple selon ses personnages plutôt que sur la base d'autres critères plus linéaires (actes ou scènes). Au-delà de la possibilité technique, une première question théorique se pose : cette unité recomposée artificiellement pour les besoins de la recherche peut-elle être considérée comme un TEXTE à part entière?

En effet, le rôle d'un personnage n'est pas un objet textuel continu, mais bien un assemblage d'interventions de nature (monologue, dialogue, aparté...) et de longueur différentes (d'un mot à plusieurs phrases) détachées de leur co(n)texte direct. La linéarité d'une œuvre de théâtre n'est donc plus strictement respectée lorsqu'elle fait l'objet d'un tel découpage. Néanmoins, les séquences réunies se succèdent dans l'ordre de la pièce ${ }^{5}$ et surtout forment ensemble une unité de sens au-delà des seules répliques strictement antérieures et postérieures : « un texte n'est pas qu'une suite linéaire de parties [...], c'est, en même temps, un tout de sens sémantico-pragmatique, une unité configurationnelle $»^{6}$. Relevons également que ces interventions sont prononcées par un même locuteur, certes fictif mais dramatiquement et significativement autonome des autres intervenants. Enfin, le rôle d'un personnage n'est jamais envisagé comme un texte indépendant à étudier de manière isolée. Il est toujours considéré comme une partie de l'ensemble global que constitue la pièce de théâtre dont il est issu. Même si la notion même de texte pourrait convenir dans une certaine mesure à un personnage de théâtre, on préfèrera utiliser l'expression « unités textuelles " plutôt que textes, ce qui évite toute confusion définitoire.

Le concept de DISCOURS semble non seulement plus pertinent, mais surtout plus fécond à notre objet d'étude. Selon E. Roulet, « le terme de discours présente le triple avantage de neutraliser la dimension écrite, de marquer nettement la différence entre les deux niveaux grammatical et discursif et de renvoyer à une unité minimale qui n'est 
plus de l'ordre de la proposition mais de l'acte $»^{7}$. Cet avantage convient d'autant plus aux œuvres de théâtre dont la question de la représentation doit absolument être prise en considération pour une juste évaluation. Plus que dans d'autres genres littéraires, le texte dramatique sert d'appui et de cadre plus ou moins souple à une performance qui peut varier d'une époque ou d'un lieu à l'autre: lecture silencieuse et individuelle aujourd'hui, lecture orale et collective sous l'empire romain, spectacle de théâtre dont les conditions de représentation ont évolué du monde grec jusqu'à nos jours. La signification des œuvres s'en trouve profondément modulée en fonction de chacune de ses réactualisations.

J.-M. Adam confirme cette position en proposant que le discours est un "énoncé caractérisable certes par des propriétés textuelles, mais surtout comme un acte de discours accompli dans une situation (participants, institution, lieu, temps)». Le contexte doit être mis en dialogue avec le texte lui-même pour analyser le discours dans tous ses aspects. La notion de discours permet donc d'envisager le rôle d'un personnage dans ses dimensions tant globales (l'œuvre) que locales (la réplique). Enfin, selon É. Benveniste, le discours permet aussi de prendre en compte l'aspect interactif (relation entre locuteur et interlocuteur) d'une production textuelle, indispensable dans un contexte dramatique ${ }^{8}$. En résumé, chacune des unités textuelles que forment les répliques d'un personnage en constitue son discours. Le discours d'un personnage est essentiellement lié à son action sur scène. Au théâtre, dire c'est souvent faire et faire c'est toujours dire, a fortiori dans le théatre ancien. Or, discours et style sont intrinsèquement liés, comme formulé par A. Herschberg: "Il en va différemment de l'approche stylistique, qui travaille sur du discours, c'est-à-dire sur du langage en situation d'énonciation $»^{9}$.

Dans ces conditions théoriques, une fois la pièce décomposée selon les discours de ses personnages, on peut ensuite mettre ces unités textuelles en comparaison au moyen des outils confirmés de la statistique textuelle et déjà utilisés sur des corpus plus larges (histogrammes, AFC, analyse arborée, cooccurrences spécifiques). Cette approche contrastive ouvre une voie d'accès d'une part aux processus de caractérisation des personnages dans leur singularité discursive (logométrie). D'autre part, elle met en lumière les dynamiques de composition d'une œuvre de théâtre par l'auteur lorsqu'on envisage un personnage au sein de l'action dramatique globale (dramaturgie) ${ }^{10}$. Par exemple, l'analyse arborée des fréquences de lemme dans l'Agamemnon de Sénèque est riche d'informations : 


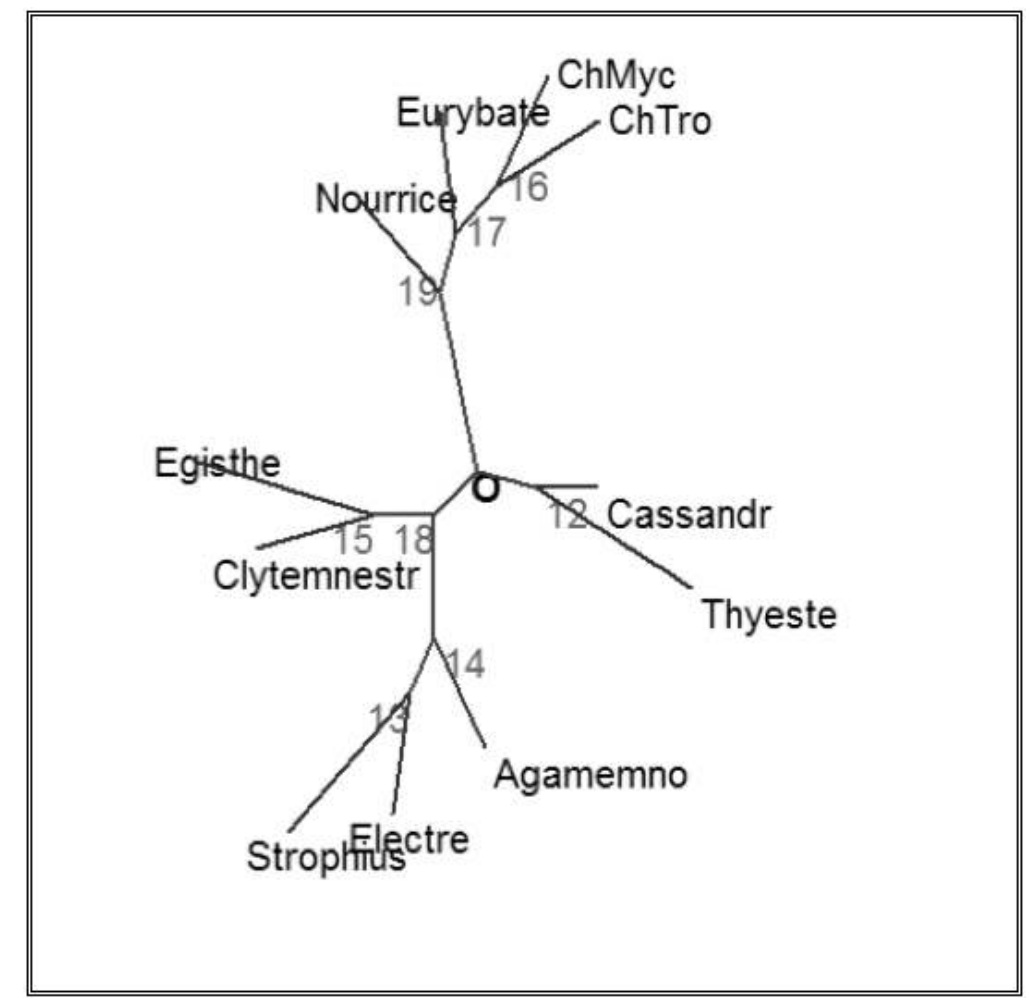

Cette pièce raconte le meurtre d'Agamemnon de retour de Troie à Mycènes par son épouse Clytemnestre et son amant Égisthe. L'analyse arborée produite ici réunit les discours des personnages certes en raison d'un facteur thématique (les personnages associés abordent les mêmes sujets), mais aussi en raison du rôle du personnage dans l'action dramatique (personnages secondaires $v s$ personnages tragiques, clans) et du type de leur discours (personnage furiosus, discours prophétique).

\section{Cette unité artificielle peut-elle avoir un style?}

La double question est désormais de savoir si la notion de style est pertinente dans le cas de ces discours et si la statistique textuelle est à même de mettre au jour des caractéristiques stylistiques spécifiques à ces unités. Au départ, le style, en littérature, était avant tout associé à un genre littéraire, une œuvre intégrale ou à un auteur en particulier ${ }^{11}$, ce qui semble a priori éloigné des personnages de théâtre. Mais, les chercheurs ont ensuite pris en compte la dimension du locuteur, déjà annoncée par le philologue J.Marouzeau, lorsqu'il écrivait que le style est «l'attitude que prend l'usager, écrivant ou parlant, vis-à-vis du matériel que la langue lui fournit $»^{12}$. Cette position est confirmée et détaillée, entre autres, par M. Riffaterre: "la stylistique étudie l'acte de communication linguistique [...] comme portant l'empreinte de la personnalité du locuteur $\aleph^{13}$. Dans une pièce, même si l'auteur est unique, le personnage occupe bien cette position de locuteur. Il défend sur scène une parole individualisée. Il adopte une attitude énonciative indépendante des autres intervenants induisant un certain degré de distance intertextuelle/interdiscursive entre chacun d'eux. Ceci a été démontré dans nos travaux précédents, mais également par d'autres chercheurs, sur Molière par exemple ${ }^{14}$. Il paraît donc pertinent de chercher maintenant 
à déterminer si les processus de caractérisation opérés par l'auteur entre les différents personnages peuvent relever dudit style.

Comme illustré par l'exemple de l'Agamemnon de Sénèque, les outils de la statistique textuelle permettent de mettre en évidence les distances entre les discours des personnages. L'analyse arborée fait apparaitre des facteurs de nature différente qu'il convient d'identifier précisément. D'abord, le facteur du mode d'énonciation apparaît nettement. Le graphique regroupe les discours en fonction d'une opposition du type monologue vs dialogue (ou locution vs interlocution), en isolant nettement les deux chœurs, le messager Eurybate et la nourrice. L'arbre sépare également des autres personnages Thyeste et Cassandre qui s'expriment tous deux dans un registre prophétique qui nécessite, dans une certaine mesure, une formulation similaire: Thyeste annonce les événements à venir au début de la pièce (par exemple, v. 47-49) et Cassandre interprète les événements en cours (v. 875-906). On peut enfin relever le facteur de la posture tragique propre à Sénèque, le furiosus qui connaît un schéma récurrent d'évolution dramatique (dolor, furor, nefas), ici Clytemnestre et Égisthe. Ces facteurs se construisent sur la base d'un vocabulaire commun. Il serait également possible de produire un graphique sur les codes grammaticaux. On pourrait donc se demander si les caractéristiques communes réunissant certains personnages entre eux sur l'arbre ne relèveraient pas du style, en tout cas dans une acception un peu large, comme défini par K. Van Dalen-Oskam: "In Digital Humanities, style is seen as anything that can be measured in the linguistic form of a text, such as vocabulary, punctuation marks, sentence length, word length, the use of character strings. [...] Every word and every feature contributes to the general outlook of the text $»^{15}$. En tout cas, on rentre sans aucun doute dans le cadre de la proposition commune de définition de J. B. Herrmann, K. van Dalen-Oskam, C. Schöch : "Style is a property of texts constituted by an ensemble of formal features which can be observed quantitatively or qualitatively ${ }^{16}$.

Dans le cas des chœurs et des messagers, il existe effectivement au-delà de la versification, une série de caractéristiques - qu'on pourrait appeler stylèmes ${ }^{17}$ - qui associées dans un même discours, confèrent un style commun auxdits personnages (par exemple: la surutilisation de l'indicatif et du participe, de la troisième personne, de l'ablatif et du nominatif). 
Figure 2. AFC des lemmes entre les 71 personnages de la base PERSONNAGES (tableau lexical entier)

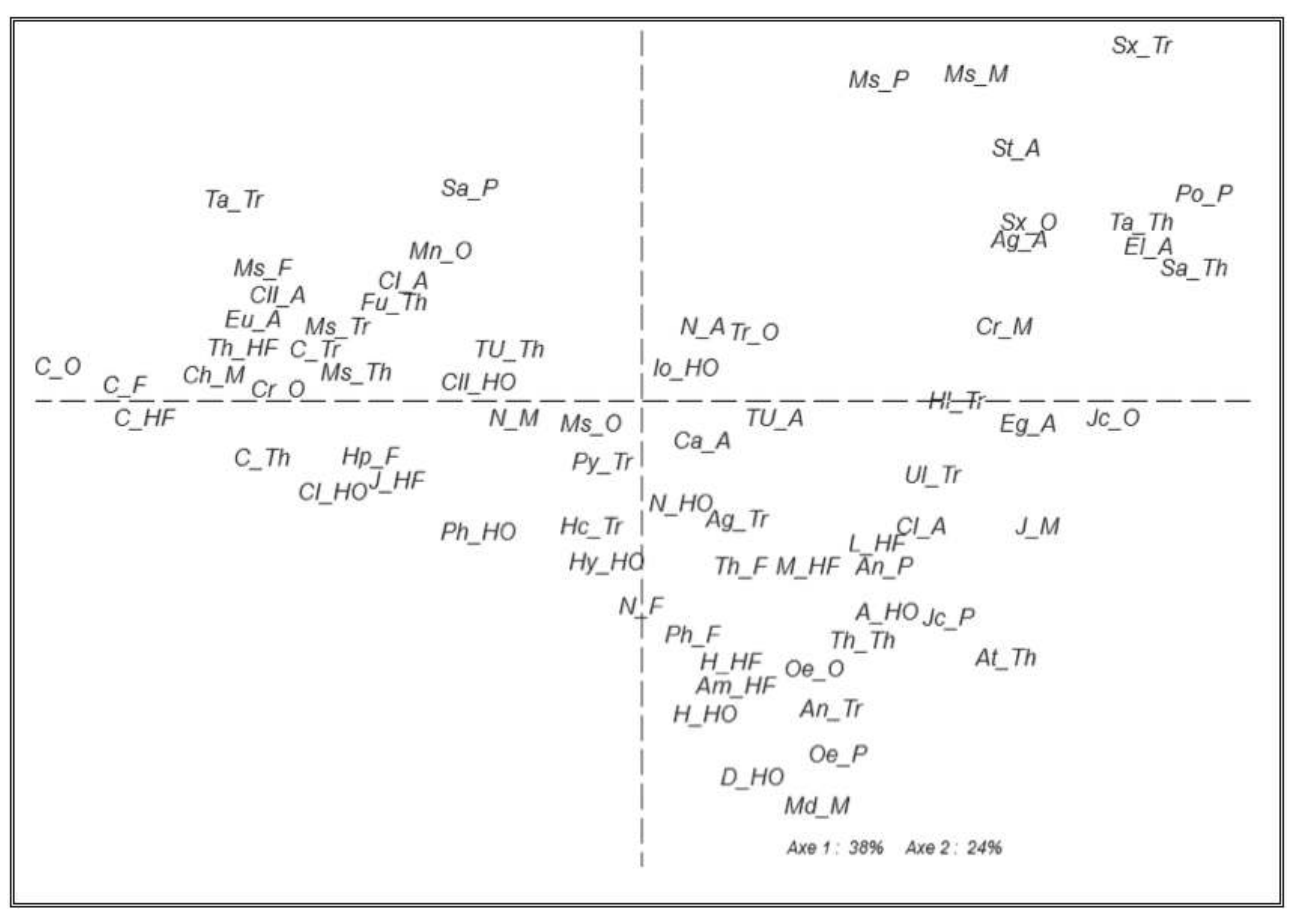

La particularité très forte de la langue de ces deux catégories de personnages a déjà été relevée et commentée sans recours à la statistique ${ }^{18}$. L'outil vient ici confirmer empiriquement les observations précédentes. Par contre, il est plus intéressant de relever que d'autres personnages de la tragédie présentent un style similaire aux messagers : Mantô de l'Édipe et Thésée de l'Hercule Furieux par exemple. Ce résultat ne s'obtient que si on élargit le corpus à l'ensemble des tragédies de Sénèque. Il nous éclaire à la fois sur le style de ces personnages, mais nous aide également à comprendre le rôle qu'ils occupent au sein de leur pièce.

Le facteur du mode de l'énonciation est également présent dans la tragédie d'Euripide et on le retrouve, même si c'est dans une moindre mesure, dans la tragédie de Corneille par exemple avec le personnage de Theudas dans Médée. Il est donc puissant au point de marquer stylistiquement le discours de personnages de pièces de langue, de culture et d'époque différentes. Dans un corpus plus large encore et qui dépasse les genres littéraires, c'est également de ce facteur dont parle F. Rastier quand il écrit que : «la représentation de l'interlocution semble partout discriminante de façon cruciale: elle singularise la littérature par rapport aux autres discours, et sépare le théâtre de la poésie et du roman $»^{19}$. Nos opérations statistiques valident donc cette analyse à un niveau beaucoup plus local d'observation.

En ce qui concerne les autres facteurs de caractérisation relevés dans l'Agamemnon, ils n'apparaissent pas dans chaque tragédie de Sénèque. Les facteurs de ce type opèrent au niveau d'une seule tragédie à la fois et ne sont pas effectifs ailleurs. En effet, l'auteur met en place des stratégies différentes de caractérisation en fonction de ses besoins dramatiques qui varient d'une pièce à l'autre. Dans les Troyennes, ce sont les personnages féminins qui présentent des caractéristiques stylistiques différentes de leurs homologues masculins dans une pièce qui met en confrontation les femmes vaincues de Troie aux guerriers grecs vainqueurs. Dans les Phéniciennes, il est plutôt question de mettre en parallèle les personnages d'ÆEdipe et de Jocaste à jamais traumatisés par le dysfonctionnement de leur famille. Pour ce faire, Sénèque développe 
un style similaire pour les époux avec un effet de répétition entre les deux grandes parties de la pièce.

Le style peut même intervenir à un niveau plus local encore qu'entre personnages. En effet, au sein d'un même discours, certains moments de l'action nécessitent un style spécifique. Par exemple, les prologues de la tragédie latine proposent un style propre à tous les personnages au-delà de leur identité, puisqu'ils partagent un vocabulaire et une morphosyntaxe communs. Chez Sénèque, ouvrir une tragédie est une procédure relativement figée tant du point de vue dramatique que discursif :

Figure 3. AFC des graphies dans la base PROL (tableau lexical entier)

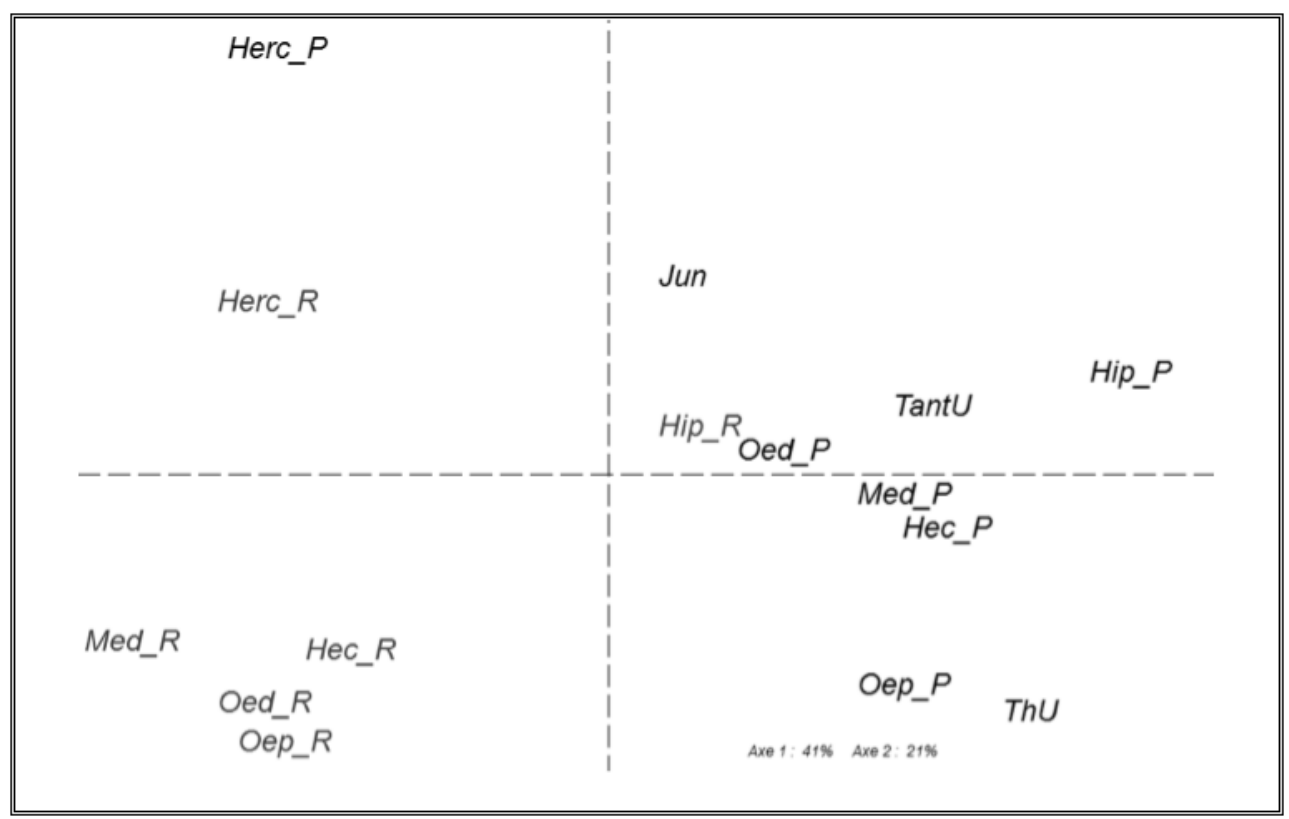

Comme nous l'avions fait pour les messagers, on notera surtout les deux exceptions : Hercule de l'Hercule sur l'Oeta présente un prologue proche de son rôle et Hippolyte de Phèdre développe un rôle similaire à son prologue. Au sein même des processus de caractérisation mis au jour - ici prologues vs rôles, au-delà de la norme de différenciation, l'écart est tout aussi significatif. Ces quelques exemples montrent à quel point la construction du corpus met en évidence des réalités stylistiques différentes et permet de faire émerger des saillances stylistiques même à un niveau très local de discours ${ }^{20}$.

\section{Style d'un seul personnage}

Par contre, les outils statistiques que nous avons utilisés (analyse arborée et AFC avec tableau entier) mettent plus difficilement au jour les caractéristiques stylistiques d'un personnage étudié individuellement. Ils sont plus efficaces pour réunir des ensembles qui partagent un certain nombre de points communs ${ }^{21}$. Par exemple, dans la Médée de Sénèque, le discours de la mère est associé par la statistique au discours de la nourrice tandis que chez Euripide et Corneille, il est rapproché du discours de Jason ${ }^{22}$. Dans le premier cas, on a montré que la nourrice se positionne comme le reflet inverse de Médée sur scène pour tenter de la dissuader d'exercer sa vengeance, comme aux vers 150-178. Dans le cas d'Euripide et de Corneille, Médée et Jason possèdent certes des caractéristiques stylistiques communes, mais il est ardu de différencier strictement ce 
qui relèverait du style de Médée de celui de Jason. Comment définir qui serait le modèle du second? De manière générale, plusieurs critères pourraient être invoqués: l'importance du personnage dans la pièce, l'ordre chronologique des répliques, le moteur de l'action dramatique, les champs lexicaux utilisés. À mon sens, il est nécessaire de revenir à l'œuvre dans sa globalité pour en comprendre ses nuances.

Si chez Sénèque, Médée est incontestablement le modèle de sa nourrice, chez Euripide et Corneille, l'exercice reste plus difficile. Il semblerait plutôt que le discours des deux personnages s'influence l'un l'autre et soit à plusieurs reprises interdépendants. Toutefois, vu le caractère hors du commun de l'héroïne, il est peu probable que son discours ne fasse pas l'objet d'une caractérisation spécifique, du moins sur certains aspects. Il convient alors de faire appel, par exemple, aux spécificités du personnage pour déterminer ce qui lui est propre (soit par la liste de spécificités soit en questionnant le corpus sur un phénomène en particulier). Chez Sénèque, le discours de Médée est caractérisé, entre autres, par une surutilisation de la première et de la deuxième personnes, particulièrement visible dans certains passages :

[MED.] TVA illa, TVA sunt illa : cui prodest scelus is fecit - omnes coniugem infamem arguant, solus TVERE, solus insontem VOCA : TIBI innocens sit quisquis est pro TE nocens.

[MÉD.] Ils sont tiens, ils sont tiens : celui à qui profite le crime l'a fait - que tous inculpent ton épouse décriée, seul défends-la, seul appelle-la innocente : que soit pour toi innocent tout qui est coupable pour ton intérêt. (Méd., 500-503)

Il s'agit bien d'une caractéristique stylistique de Médée qui cherche à rappeler les sphères de ses interlocuteurs, soit pour s'opposer à eux (Créon), soit pour essayer de les rallier à sa cause (nourrice, Égée), sans qu'il ne soit toujours possible de faire la distinction (Jason). En tout état de cause, cet effet provoque une distance entre elle et les autres.

On pourrait parler ici de trait de style au sens de G. Mathis ${ }^{23}$ en raison de sa récurrence tout au long de l'œuvre et face à différents interlocuteurs. Cet exemple rapide démontre qu'il est nécessaire de multiplier les opérations statistiques sur le corpus pour faire émerger non seulement les tendances générales mais également les caractéristiques plus ciblées. En ce qui concerne le personnage de Médée, il est

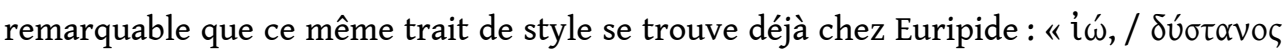

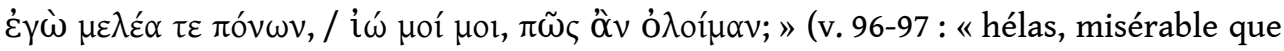
je suis, malheureuse de mes peines; hélas, pauvre de moi, de moi, comment pourrais-je mourir ?» $)^{24}$; mais aussi qu'il apparait étonnamment encore chez Corneille :

[Médée] Tous vos héros enfin tiennent de moi la vie,

Je vous les verrai tous posséder sans envie,

Je vous les ai sauvés, je vous les cède tous;

Je n'en veux qu'un pour moi, n'en soyez point jaloux. (Méd., v. 437-440)

L'extrait illustre l'opposition entre Médée et les autres ici encore accentuée par l'anaphore « je vous les » répétée trois fois.

La surutilisation des première et deuxième personnes se présente donc comme un trait de style associé au personnage de Médée par les dramaturges d'époque, de la culture et de langue différente. Il semblerait que la compréhension même du personnage de Médée par les trois auteurs induise certaines similarités dans la caractérisation de son discours sur scène ${ }^{25}$ : la mère est exclue de la société dans laquelle elle évolue et ceci modifie ses pratiques d'interaction avec les autres. Ce résultat démontre que le caractère générique de Médée, héroïne mythologique intemporelle, influence le style de son discours. Si celui-ci n'est pas isolé par les outils statistiques d'analyse globale, il n'en possède pas moins des caractéristiques stylistiques propres qui le distinguent des 
autres personnages. Ces spécificités ne sont toutefois pas suffisamment nombreuses, dans ce cas-ci pour donner au discours un style totalement indépendant des autres personnages. À nouveau, il n'est possible de déceler ce type de variation qu'en comparant les trois versions de cette tragédie - soit en modulant le corpus de référence - et en abordant le corpus avec plusieurs outils statistiques différents. Le discours d'un personnage de théatre peut donc tout à fait présenter un style, ou du moins des traits de style, qui lui sont propres au sens de N. Garric et H. Maurel-Indart :

En parlant de style, nous ne faisons pas référence à l'empreinte singulière d'un auteur - encore que la notion d'auctorialité soit liée à celle de style - ou, en d'autres termes, à une appropriation exclusivement individuelle et esthétique de la langue, mais à des spécificités textuelles plus ou moins stables, observables en corpus et construites par lui. Cette conception du style ne se confond pas avec un ornement caractéristique de la littérarité : elle est afférente à tout texte dans lequel le style se réalise en vue d'une finalité et en fonction d'un certain nombre de déterminations portées par la notion de discours ${ }^{26}$.

\section{5. Études du discours et stylistique}

Nos observations soulèvent également la relation étroite mais complexe qui existe entre études du discours et stylistique, deux notions elles-mêmes souvent polysémiques. Le rapport entre ces deux disciplines n'est pas facile à définir et suscite la perplexité des chercheurs ${ }^{27}$. Une série de points communs les unit, mais participe aussi à la confusion entre les deux disciplines tant d'un point de vue théorique qu'institutionnel ${ }^{28}$ : toutes deux peuvent travailler sur les mêmes supports, à savoir les textes; elles peuvent avoir le même objet d'étude, à savoir des discours et peuvent avoir recours aux mêmes outils heuristiques, par exemple la statistique textuelle ${ }^{29}$. L'application du concept de style aux discours de personnage de théâtre pourrait, me semble-t-il, apporter très modestement une pierre à l'édifice. Elle a fait apparaître que la priorité des objectifs poursuivis n'est pas exactement identique. Certaines pratiques des chercheurs des deux domaines sont donc similaires, mais leur rapport à la matière en est modifié.

Il me semble que les analystes cherchent d'abord à comprendre POURQUOI un texte, en tant qu'acte de langage (locuteur 惯 interlocuteur), fait l'objet d'un processus de caractérisation par son auteur. Il l'étudie ainsi en tant que discours intégré à un genre, lui-même faisant partie d'un inter-discours plus large. Pour atteindre son objectif, il doit aussi aborder le discours comme un objet textuel construit à appréhender d'un niveau global au plus local. Pour ce faire, il fait appel à toutes les disciplines qu'il juge utile: textométrie, lexicologie, sémantique, linguistique, rhétorique, poétique. Le stylisticien me paraît plus intéressé à analyser COMMENT ce même texte a fait l'objet d'un processus de caractérisation. Il se concentre plutôt sur les variations que subit le texte en tant qu'objet concret. Évidemment, se poser l'une ou l'autre question amène inévitablement à répondre, du moins en partie, à la seconde ce qui rend complexe la délimitation d'une frontière nette entre les deux champs d'études. Toutefois, il me semble plus fécond de clarifier les ponts et de prendre conscience que l'ont fait de la stylistique en analyse du discours et de l'analyse du discours en stylistique que de chercher à tout prix à dissocier les deux approches, l'une enrichissant incontestablement l'autre. 


\section{Conclusions}

À la fin de ce parcours au sein du théâtre, on peut tirer plusieurs conclusions. En premier lieu, il est apparu que les discours de personnage de théâtre, unités textuelles recomposées artificiellement par le chercheur, peuvent faire l'objet d'une recherche d'ordre stylistique. Les outils de la statistique textuelle permettent de faire émerger des réalités stylistiques de nature et de degré divers en fonction du niveau de caractérisation : style de catégorie générique de personnage (messager ou chœur), style entre personnages d'une même pièce (le couple Clytemnestre-Égisthe), style ou trait stylistique d'un seul personnage, style d'un moment précis de l'action (les prologues). Sur ce point, nous rejoignons la conclusion J.-M. Adam sur la stylistique et l'analyse textuelle: "Le style est partout dans le texte, à tous les niveaux de construction de sens ${ }^{30}$. Nos observations montrent par contre que chaque personnage ne fait l'objet d'une caractérisation qui le rendrait indépendant stylistiquement des autres personnages. Le processus est mis en place de manière plus ou moins puissante selon les besoins de l'auteur.

En deuxième lieu, et en corolaire de ce qui vient d'être dit, il est apparu que le style d'un personnage de théâtre ne dépend pas que du locuteur. Il peut être généré par un ou plusieurs facteurs de natures différentes (locuteur, posture d'énonciation, type de discours...). Il est donc important d'identifier ces facteurs, de les définir et de les analyser en détail pour comprendre les processus stylistiques en vigueur dans chaque texte et pour repérer tous les facteurs générateurs de style. Le style n'est donc plus limité uniquement à l'auteur, ni même au locuteur. Il se comprend désormais comme l'ensemble des variations au sein d'un corpus prédéterminé. Il se veut donc pluriel et multifactoriel.

En troisième lieu, un apport important relève également de la méthodologie. La place du corpus semble prépondérante dans notre rapport au style puisque ce sont ses différentes configurations qui ont fait émerger le style des sous-corpus. Cet aspect a évidemment déjà été mis en évidence ${ }^{31}$, mais j'aimerais faire un pas supplémentaire en proposant que le corpus lui-même doit être considéré comme un outil de recherche et être exploité en ce sens. Par sa définition et son partitionnement, le corpus aidé des outils statistiques, est un opérateur de contraste puissant et c'est par le contraste même que le style est mis en lumière. Afin de faire apparaître tous les facteurs de caractérisation, il est donc nécessaire de multiplier les découpages du corpus de référence et de procéder à ce que nous avons appelé précédemment, une lecture polyangulaire du texte ${ }^{32}$. C'est de cette manière qu'il est possible d'appréhender aussi précisément que possible toutes les nuances stylistiques en jeu au sein du corpus de base.

\section{BIBLIOGRAPHIE}


Adam J.-M. (2001). « Types de textes ou genres de discours ? Comment classer les textes qui disent de et comment faire?", Langages $141: 10-27$.

Adam J.-M. (2005). « Stylistique ou analyse textuelle ? L'exemple du fragment 128 des Caractères », dans Gouvard J.-M. (éd.), De la langue au style. Lyon : Presses Universitaires de Lyon, 127-144.

Amossy R. \& Maingueneau D. (éd.) (2003). L'analyse du discours dans les études littéraires. Toulouse : Presses Universitaires du Mirail.

Baertschi A. M. (2010). « Drama and Epic Narrative : The Test Case of Messenger Speech in Seneca's Agamemnon ", dans Gildenhard I. et Revermann M. (éd.). Beyond the Fifth Century: Interaction with Greek Tragedy from the Fourth Century BCE to the Middle Ages. Berlin : Walter de Gruyter, 249-268.

Benveniste É. (1970). « L'appareil formel de l'énonciation », Langages 17, p. 12-18.

Bernard M. « Spécificités lexicales d'un sous-corpus : quel(s) corpus de référence ? ", dans Garric N. et Maurel-Indart H. (dir.). Vers une automatisation de l'analyse textuelle, dans Texto !, [En ligne], vol. XV, $\mathrm{n}^{\circ} 4$ (2010) et XVI, $\mathrm{n}^{\circ} 1$ (2011), 33-36.

Brunet É. (2011). HYPERBASE : Manuel de référence, première partie. Nice.

Burrows J. (2002). « Delta : a Measure of Stylistic Difference and a Guide to Likely Authoship », Literary and Linguistic Computing 17 : 267-287.

Fantoli M. \& Vandersmissen M. (2019). « Partitionnements multiples de corpus : une lecture polyangulaire? L'exemple des bases latines du LASLA », Corpus 19 [en ligne].

Frontini F., Boukhaled M.A. \& Ganascia J.-F. (2015). « Moliere's Raisonneurs: a Quantitative Study of Distinctive Linguistic Patterns », Corpus Linguistics, Lancaster, United Kingdom.

Garelli-François M.-H. (1998). « Tradition littéraire et création dramatique dans les tragédies de Sénèque : l'exemple des récits de messagers ", Latomus $57:$ 15-32.

Garric N. \& Maurel-Indart H. (dir.). Vers une automatisation de l'analyse textuelle, dans Texto !, [En ligne], vol. XV, $\mathrm{n}^{\circ} 4$ (2010) et XVI, $\mathrm{n}^{\circ} 1$ (2011).

Guyot A. (2006). « Stylèmes et corpus génériques : un essai de confrontation au service de la stylistique des genres », Corpus 5 [En ligne].

Harris Z. S. \& Dubois-Charlier F. (1969). « Analyse du discours », Langages 13 : 8-45.

Herrmann J. B., van Dalen-Oskam K. \& Schöch C. (2015). « Revisiting Style, a Key Concept in Literary Studies ", Journal of Literary Theory $9: 25-52$.

Herschberg Pierrot A. (2006). «Style, corpus et genèse », Corpus 5 [En ligne].

Jacquot C. (2016). « Rêve d'une épiphanie du style : visibilité en stylistique et en stylométrie », Revue d'histoire littéraire en France 3 : 619-770.

Magri-Mourgues V. (2006). « Corpus et stylistique », Corpus 5 [en ligne].

Maingueneau D. (2008). «Stylistique, analyse du discours littéraire », dans Durand J., Habert B. \& Laks B. (éd.) Congrès Mondial de Linguistique Française - CMLF'08. Paris : Institut de Linguistique Française.

MAINGUENEAU D. (2011). « Linguistique, littérature, discours littéraire », Le français aujourd'hui $175: 75-82$.

Marouzeau J. (1970). Traité de stylistique latine. Paris : Les Belles Lettres.

Mathis G. (1997). « Stylistique et discours scientifique », ASp 15-18 [En ligne]. 
Mellet S. \& Longrée D. (2014). « Les variantes des motifs chez les prosateurs latins : entre récurrence générique et spécificité d'auteur, des formes révélatrices et caractérisantes ", LEC 82 : 65-87 [en ligne].

Meschonnic H. (1970). Pour la poétique. Paris : Gallimard, 1970.

Paveau M.-A. (2011). L'analyse linguistique du texte littéraire. Une fausse évidence. Le Français Aujourd'hui. Armand Colin - Dunod, 83-94.

Petit M. (1997). « Stylistique(s) contrastive(s) du discours scientifique », ASp 15-18 : 139-156.

Petitjean A. (2010). « Pour une stylistique des œuvres dramatiques : l'exemple des didascalies », Stylistiques? [en ligne]. Rennes : Presses universitaires de Rennes.

Rastier F. (2011). La mesure et le grain : Sémantique de corpus. Paris : Honoré Champion, coll. « Lettres numériques $12 »$.

Riffaterre M. (1971). Essais de stylistique structurale. Paris : Flammarion.

Roulet E. (1991). « Une approche discursive de l'hétérogénéité discursive », ELA 83 : 123.

Van Dalen-Oskam K. (2012). De stijl van R. Inaugural lecture. Amsterdam, http://

www.oratiereeks.nl/upload/pdf/PDF-1290Weboratie_Van_Dalen-Oskam.pdf.

Vandersmissen M. (2019). Le discours des personnages féminins chez Sénèque : approches logométriques et contrastives d'un corpus théâtral. Bruxelles : Latomus, « Collection Latomus 359 ».

Vandersmissen M. (2020). « Discours de Médée : Logométrie et Médée d'Euripide », dans De la Escosura Balbas M. C., Duce Pastor E., González Gutierrez P., Rodriguez Alcocer M. M. et Serrano Lozano D. (éd.), Blame it on the Gender : Identities and Transgressions in Antiquity. Oxford: BAR Publishing, 41-51.

Vandersmissen M. (2021). « Discours masculins et féminins dans la Médée de Corneille : un modèle sénéquéen? ", dans Charlet-Mesdjian B. et Flicker C. (éd.) Seneca Tragicvs : Vir, Vis, Violentia, Virtus, Virago : La virilité et ses déclinaisons dans le théâtre de Sénèque et chez ses émules de Mussato à nos jours: actes du colloque des 27-29 mars 2019 à l'Université Aix-Marseille. Marseille : Presses Universitaires de Provence (sous presse).

\section{NOTES}

1. Magri-Mourgues V. (2006). « Corpus et stylistique », Corpus 5 [en ligne].

2. Petitjean A. (2010). « Pour une stylistique des œuvres dramatiques : l'exemple des didascalies", Stylistiques? [en ligne]. Rennes: Presses universitaires de Rennes; Petit M. (1997). "Stylistique(s) contrastive(s) du discours scientifique », ASp 15-18, 139-156.

3. Guyot A. (2006). «Stylèmes et corpus génériques : un essai de confrontation au service de la stylistique des genres», Corpus 5 [En ligne]; Mellet S. \& Longrée D. (2014). "Les variantes des motifs chez les prosateurs latins : entre récurrence générique et spécificité d'auteur, des formes révélatrices et caractérisantes ", LEC 82 [en ligne], 65-87.

4. Nous travaillons sur les fichiers grecs et latins annotés par le LASLA de l'Université de Liège et principalement avec le logiciel Hyperbase CD-ROM ou Web édition (http://hyperbase.unice.fr/). Voir : Brunet É. (2011). HYPERBASE : Manuel de référence, première partie. Nice.

5. Harris Z. S. \& Dubois-Charlier F. (1969). « Analyse du discours », Langages 13 : 24-25 : « Le texte peut être constitué de morceaux successifs, sortes de sous-textes à l'intérieur du texte principal, comme des paragraphes ou des chapitres ". 
6. Adam J.-M. (2001). « Types de textes ou genres de discours? Comment classer les textes qui disent de et comment faire? ", Langages $141: 19$.

7. Roulet E. (1991). « Une approche discursive de l'hétérogénéité discursive », ELA $83: 123$.

8. Benveniste É. (1970). «L'appareil formel de l'énonciation », Langages $17: 14$.

9. Herschberg Pierrot A. (2006). «Style, corpus et genèse », Corpus 5 [En ligne].

10. Vandersmissen M. (2019). Le discours des personnages féminins chez Sénèque: approches logométriques et contrastives d'un corpus théâtral. Bruxelles : Latomus, « Collection Latomus 359 ».

11. "C'est l'œuvre qui fait le style» écrivait M. Meschonnic dans Pour la Poétique (p. 20); voir aussi les travaux de reconnaissance d'auteur, par exemple : Burrows J. (2002). « Delta : a Measure of Stylistic Difference and a Guide to Likely Authoship », Literary and Linguistic Computing 17 : 267-287.

12. Marouzeau J. (1970). Traité de stylistique latine, p. xiii.

13. Riffaterre M. (1971). Essais de stylistique structurale. Paris : Flammarion, 145.

14. Frontini F., Boukhaled M.A. \& Ganascia J.-F. (2015). «Moliere's Raisonneurs: a Quantitative Study of Distinctive Linguistic Patterns », Corpus Linguistics, Lancaster, United Kingdom.

15. Van Dalen-Oskam K. (2012). De stijl van R. Inaugural lecture, Amsterdam, http:// www.oratiereeks.nl/upload/pdf/PDF-1290Weboratie_Van_Dalen-Oskam.pdf.

16. Herrmann J. B., van Dalen-Oskam K. \& Schöch C. (2015). « Revisiting Style, a Key Concept in Literary Studies ", Journal of Literary Theory $9: 44$.

17. Jacquot C. (2016). «Rêve d'une épiphanie du style : visibilité en stylistique et en stylométrie », Revue d'histoire littéraire en France 3 : 625-627.

18. Baertschi A. M. (2010). «Drama and Epic Narrative: The Test Case of Messenger Speech in Seneca's Agamemnon » dans Gildenhard I. \& Revermann M. (éd.), 249-268 ; Garelli-François M.-H. (1998). « Tradition littéraire et création dramatique dans les tragédies de Sénèque : l'exemple des récits de messagers ", Latomus $57: 15-32$.

19. Rastier F. (2011), p. 92-95.

20. Magri-Mourgues V. (2006). « Corpus et stylistique », Corpus $5: 1$ [en ligne].

21. Comme on l'a vu avec les messagers ou les prologues, des exceptions peuvent émerger de ces ensembles. Elles sont tout à fait pertinentes dans pour la recherche stylistique mais sont le fruit d'une procédure empirique.

22. Vandersmissen M. (2021). "Discours masculins et féminins dans la Médée de Corneille : un modèle sénéquéen?", dans Charlet-Mesdjian B. et Flicker C. (éd.) Seneca Tragicvs: Vir, Vis, Violentia, Virtus, Virago: La virilité et ses déclinaisons dans le théâtre de Sénèque et chez ses émules de Mussato à nos jours: actes du colloque des 27-29 mars 2019 à l'Université Aix-Marseille, Marseille : Presses Universitaires de Provence (sous presse).

23. Mathis G. (1997). « Stylistique et discours scientifique », ASp 15-18: 5 [En ligne].

24. Vandersmissen M. (2020). «Discours de Médée : Logométrie et Médée d'Euripide », dans De la Escosura Balbas M. C., Duce Pastor E., González Gutierrez P., Rodriguez Alcocer M. M. et Serrano Lozano D. (éd.), Blame it on the Gender: Identities and Transgressions in Antiquity, Oxford: BAR Publishing, 2020, p. 46.

25. L'influence d'un auteur sur les autres pourrait aussi être évoquée.

26. Garric N. \& Maurel-Indart H. (dir.). Vers une automatisation de l'analyse textuelle, dans Texto!, [En ligne], vol. XV, $n^{\circ} 4$ (2010) et XVI, $n^{\circ} 1$ (2011), p. 9.

27. Voir le chapitre IV, "Analyse du discours et style» de l'ouvrage réunissant trois communications: Amossy R. \& Maingueneau D. (éd.) (2003). L'analyse du discours dans les études littéraires, Toulouse : Presses Universitaires du Mirail.

28. Maingueneau D. (2008). "Stylistique, analyse du discours littéraire", dans Durand J., Habert B., Laks B. (éd.), Congrès Mondial de Linguistique Française - CMLF'08, Paris, Institut de Linguistique Française ; MAINGUENEAU D. (2011). « Linguistique, littérature, discours littéraire », Le français aujourd'hui $175: 75-82$. 
29. À titre d'exemple, on peut citer : Bernard M., « Spécificités lexicales d'un sous-corpus : quel(s) corpus de référence?", dans Garric N. et Maurel-Indart H. (dir.). Vers une automatisation de l'analyse textuelle, dans Texto !, [En ligne], vol. XV, $\mathrm{n}^{\circ} 4$ (2010) et XVI, $\mathrm{n}^{\circ} 1$ (2011), p. 33-36.

30. Adam J.-M. (2005). "Stylistique ou analyse textuelle? L'exemple du fragment 128 des Caractères ", dans Gouvard J.-M. (éd.), De la langue au style, Lyon : Presses Universitaires de Lyon, p. 141.

31. Voir le volume 5 (2006) de la revue Corpus, « Corpus et stylistique ».

32. Fantoli M. \& Vandersmissen M. (2019). «Partitionnements multiples de corpus : une lecture polyangulaire? L'exemple des bases latines du LASLA », Corpus 19 [en ligne].

\section{RÉSUMÉS}

Dans le cadre du développement récent de la stylistique outillée, cet article propose une réflexion sur l'application de ce concept et de ses méthodes aux personnages de théâtre sur la base d'un corpus trilingue de tragédies: Euripide, Sénèque et Corneille. Pour mener la recherche, nous aborderons d'abord la question de la nature des rôles de théâtre entre unités textuelles recomposées et discours de personnages dans le cadre d'une performance sur scène. Ensuite, nous chercherons à définir si les caractéristiques de ces unités peuvent être rapprochées de la notion de style. Nous en étudierons les apports pour la compréhension d'une œuvre de théâtre. Pour atteindre cet objectif, une attention particulière sera accordée au corpus de recherche (quelles œuvres choisir?), à sa constitution (comment diviser ce corpus et avec quel niveau de granularité ?) et aux outils d'exploration (qualitative ou quantitative ?). En stylistique outillée, le corpus devient lui-même un outil de recherche en tant qu'opérateur de contraste. Enfin, poursuivre cette enquête amène à questionner la frontière entre études du discours et stylistique outillée du point de vue de leurs objectifs et de leurs méthodes.

In the context of the recent development of digital stylistics, this paper proposes a study on the application of this concept and its methods to theatrical characters on the basis of a trilingual corpus of tragedies: Euripides, Seneca and Corneille. To conduct the research, we will first address the question of the nature of theatrical roles between recomposed textual units and character discourse in the context of a stage performance. Then, we will try to define if the characteristics of these units can be related to the notion of style. We will study its contribution to the understanding of a work of theatre. To achieve this objective, particular attention will be paid to the research corpus (which works should be chosen?), to its creation (how should this corpus be divided and with what level of granularity?) and to the exploration tools (qualitative or quantitative?). In digital stylistic, the corpus itself becomes a research tool as a contrast operator. Finally, pursuing this investigation leads us to question the frontier between discourse studies and digital stylistics in terms of their objectives and methods.

\section{INDEX}

Mots-clés : stylistique outillée, études du discours, statistique textuelle, personnages de théâtre Keywords : digital stylistics, discourse studies, textual statistics, theatre characters 
AUTEUR

MARC VANDERSMISSEN

Université de Liège - LASLA

mvandersmissen@uliege.be 